\title{
ÁRBOLES DE FORZAMIENTO SEMÁNTICO PARA OPERACIONES ENTRE CONJUNTOS
}

\section{SEMANTIC FORCING TREES FOR OPERATIONS BETWEEN SETS}

\author{
Manuel SIERRA-ARISTIZÁBAL ${ }^{1}$ \\ ${ }^{1}$ Departamento de Ciencias Matemáticas, Universidad EAFIT. \\ *Autor Corresponsal. E-mail: msierra@eafit.edu.co
}

\begin{abstract}
Historia del Artículo
Recibido: Agosto 28, 2016

Evaluado: Enero 08, 2016

Aceptado: Enero 15, 2017

Disponible: Febrero 09, 2017
\end{abstract}

\section{Resumen |}

\begin{abstract}
La semántica de valoraciones para la lógica que gobierna las operaciones entre conjuntos es caracterizada por una herramienta de inferencia visual llamada árboles de forzamiento semántico para operaciones entre conjuntos. Dada una fórmula, con esta herramienta se marcan los nodos del árbol asociado a la misma, y se determina si la fórmula es válida o no. En el caso que la fórmula sea inválida, la valoración que la refuta está determinada por las marcas de las hojas en su árbol de forzamiento, además, con esta valoración se construyen los conjuntos que generan el contraejemplo. El análisis de validez realizado con los árboles de forzamiento semántico para operaciones entre conjuntos, evita el paso por los árboles de forzamiento para la lógica de predicados, por lo que las pruebas son más cortas y más intuitivas.
\end{abstract}

Palabras Clave: Árbol de forzamiento, valoración, semántica, conjunto.

\section{Abstract}

The semantics of valuations for the logic that governs the operations between sets is characterized by a visual inference tool called: semantic forcing trees for operations between sets. Given a formula, involving this tool, the associated nodes to the tree are marked, and determine whether the formula is valid or not. If the formula is invalid, the refuting valuation is determined by the marks of the leaves in its forcing tree. The sets generate the counterexample, are then constructed with this valuation. The validity analysis performed with the semantic forcing trees for operations between sets, avoids the passage through the trees of forcing for the predicate logic, so the tests are shorter and more intuitive.

Keywords: Forcing tree, valuation, semantics, set.

\section{INTRODUCCIÓN |}

Beth (1962), presenta el método de las tablas semánticas, el cual posteriormente es popularizado por Smullyan (1994), como árboles de opciones semánticas. El método consiste en examinar sistemáticamente, todas las opciones que podrían hacer falsa una proposición dada, y buscar si una de estas opciones es lógicamente posible al no generar contradicciones, en éste caso se tiene un contraejemplo que refuta la validez de la proposición dada. Si el contraejemplo no puede ser generado, es decir ninguna opción resulta lógicamente posible, entonces la proposición analizada es válida. Este método ha sido ampliamente aceptado, y como hacen (Carnielli, 1987; Barrero y Carnielli, 2005), las tablas semánticas se han extendido a muchos sistemas de lógicas no clásicas, siendo además fácil de implementar con un programa de computador.

Los árboles de forzamiento semántico presentados por (Sierra, 2001; Sierra, 2006), no exploran todas las opciones posibles cuando se busca el contraejemplo, como se hace con las tablas semánticas, sino que, los árboles de forzamiento se limitan a las opciones que son deductivamente forzadas por las reglas del sistema. Lo anterior implica, que el análisis de validez con los árboles de forzamiento es más y natural y menos complejo que con las tablas semánticas.

Como una extensión de los árboles de forzamiento, en este trabajo se presentan los Árboles de Forzamiento Semántico para Operaciones entre Conjuntos. Se prueba con todo detalle la equivalencia entre la presentación con 
árboles de forzamiento y la presentación semántica con valoraciones. Finalmente, se muestra que si el árbol de una fórmula está bien marcado, lo cual significa que la fórmula es inválida, entonces la lectura de las marcas de las fórmulas atómicas, proporciona una valoración que refuta la validez de la fórmula, y además, con esta valoración se construyen los conjuntos que la refutan, es decir, los conjuntos que generan el contraejemplo.

\section{LENGUAJE DE LA LÓGICA DE CONJUNTOS}

Ampliando el lenguaje del cálculo proposicional clásico presentado en Caicedo (1990), el lenguaje de la Lógica para conjuntos, LC, consta de los conectivos binarios $\rightarrow, \wedge, \vee y \leftrightarrow$, de los operadores binarios $\cup, \in \mathrm{y} \subseteq$ (unión, pertenencia e inclusión de conjuntos), del conectivo monádico $\sim$, del operador monádico * (complemento de conjuntos), además del paréntesis izquierdo y el paréntesis derecho. También se tiene una cantidad enumerable de variables, constantes $y$ conjuntos primitivos. El conjunto de fórmulas y de conjuntos de LC es generado por las siguientes reglas y sólo por ellas:

Las constantes $U$ y $\varnothing$ son conjuntos primitivos.

Todo conjunto primitivo es un conjunto.

Si $\mathrm{X}$ es un conjunto entonces $(\mathrm{X})^{*}$ es un conjunto.

Si $X$ y $Y$ son conjuntos entonces $(X) \cup(Y)$ es un conjunto.

Si $X$ es un conjunto primitivo y $z$ es una variable o una constante entonces $\mathrm{z} \in \mathrm{X}$ es una fórmula atómica.

Toda fórmula atómica es una fórmula.

Si $X$ es un conjunto y $z$ es una variable o una constante entonces $z \in X$ es una fórmula.

Si $X$ y $Y$ son conjuntos entonces $X \subseteq Y$ es una fórmula.

Si A es una fórmula entonces $\sim(A)$ es una fórmula.

Si $A$ y $B$ son fórmulas entonces $(A) \wedge(B),(A) \vee(B)$, $(A) \rightarrow(B)$ y $(A) \leftrightarrow$ (B) son fórmulas.
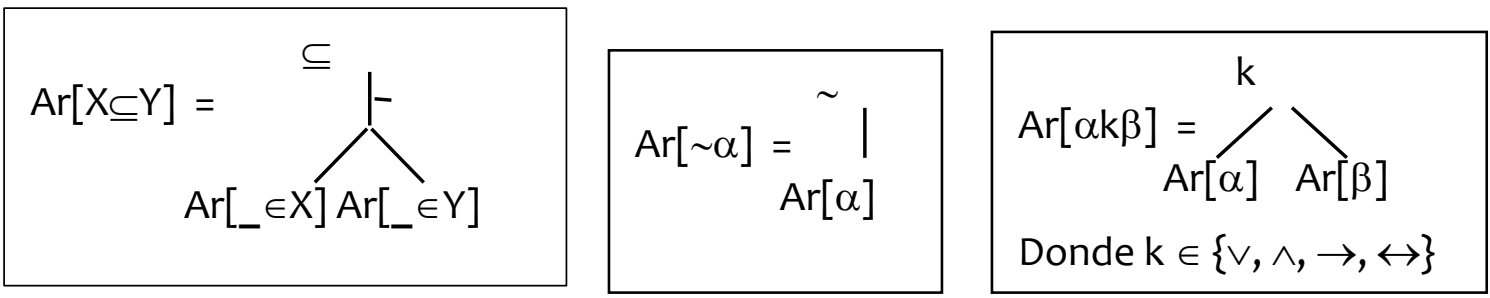

\section{ÁRBOL DE UNA FÓRMULA |}

Sea $\alpha$ una fórmula en la cual no figuran variables de individuo, el árbol inicial de $\alpha$ se representa por $\operatorname{Ar}[\alpha]$ y se construye utilizando las reglas que se presentan en la Fig. 1 ( $\alpha$ y $\beta$ fórmulas arbitrarias, $X$ y $Y$ conjuntos arbitrarios).

La señal, “_”, es el espacio vacío generado por el nodo k, el cual a su vez es el espacio vacío asociado a los hijos del nodo $\mathrm{k}$ y a los hijos de los hijos, etc. La señal " $t$ " puede ser una constante o el espacio vacío “_".

Se define el árbol inicial del argumento 'de $\alpha_{1}, \ldots, \alpha_{\mathrm{n}}$ se infiere $\beta^{\prime}$ como: $\operatorname{Ar}\left[\left(\alpha_{1} \wedge \ldots \wedge \alpha_{n}\right) \rightarrow \beta\right]$. El nodo superior del árbol de la fórmula $\alpha$, es llamado la raíz del árbol, se denota $\mathrm{R}[\alpha]$ y corresponde al operador principal de la fórmula $\alpha$. Los nodos inferiores, es decir aquellos de los cuales no salen ramas, son llamados hojas y corresponden a las fórmulas atómicas.

Por ejemplo, para el argumento: 'de $X^{*} \subseteq Z$ y $Y \subseteq \varnothing$ se infiere $\sim(X \subseteq Y \cup Z)$ ', el condicional asociado es $\left(X^{*} \subseteq Z \wedge Y \subseteq \varnothing\right)$ $\rightarrow \sim(\mathrm{X} \subseteq \mathrm{Y} \cup \mathrm{Z})$ y su árbol se presenta en la Fig. 2.

\section{MARCANDO LOS NODOS DE UN ÁRBOL}

Si un nodo $C$ es el conectivo monádico, $\sim$, o el operador monádico, *, entonces su único hijo se llama el alcance del operador y para hacer referencia a él se utiliza la notación $a C$.

Si un nodo $K$ es uno de los operadores binarios $\subseteq$ o $\cup$, o uno de los conectivos binarios $\wedge, \vee, \rightarrow 0 \leftrightarrow$, entonces para sus hijos izquierdo y derecho se utiliza la notación iK y $d K$ respectivamente.

Para toda fórmula $\beta$, el nodo asociado a $\beta$ es la raíz de $\beta$, $R[B]$, la cual a su vez es el operador principal de $\beta$ en el caso que $\beta$ sea compuesta, o es la misma $\beta$ en el caso que $\beta$ sea atómica.
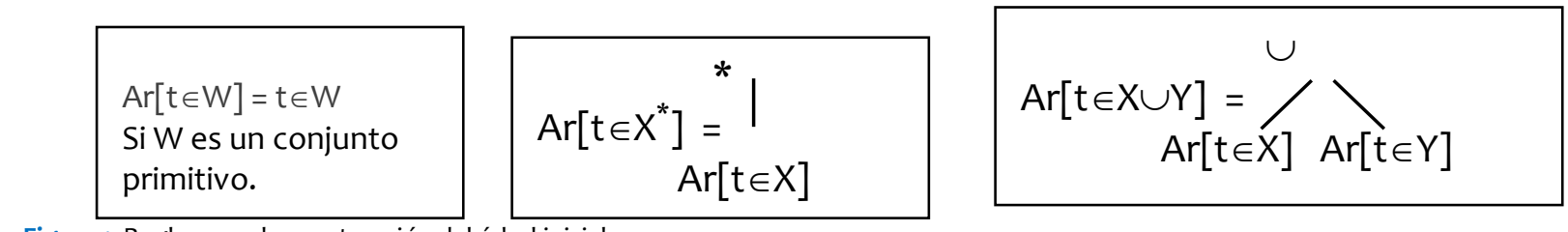

Figura 1. Reglas para la construcción del árbol inicial. 


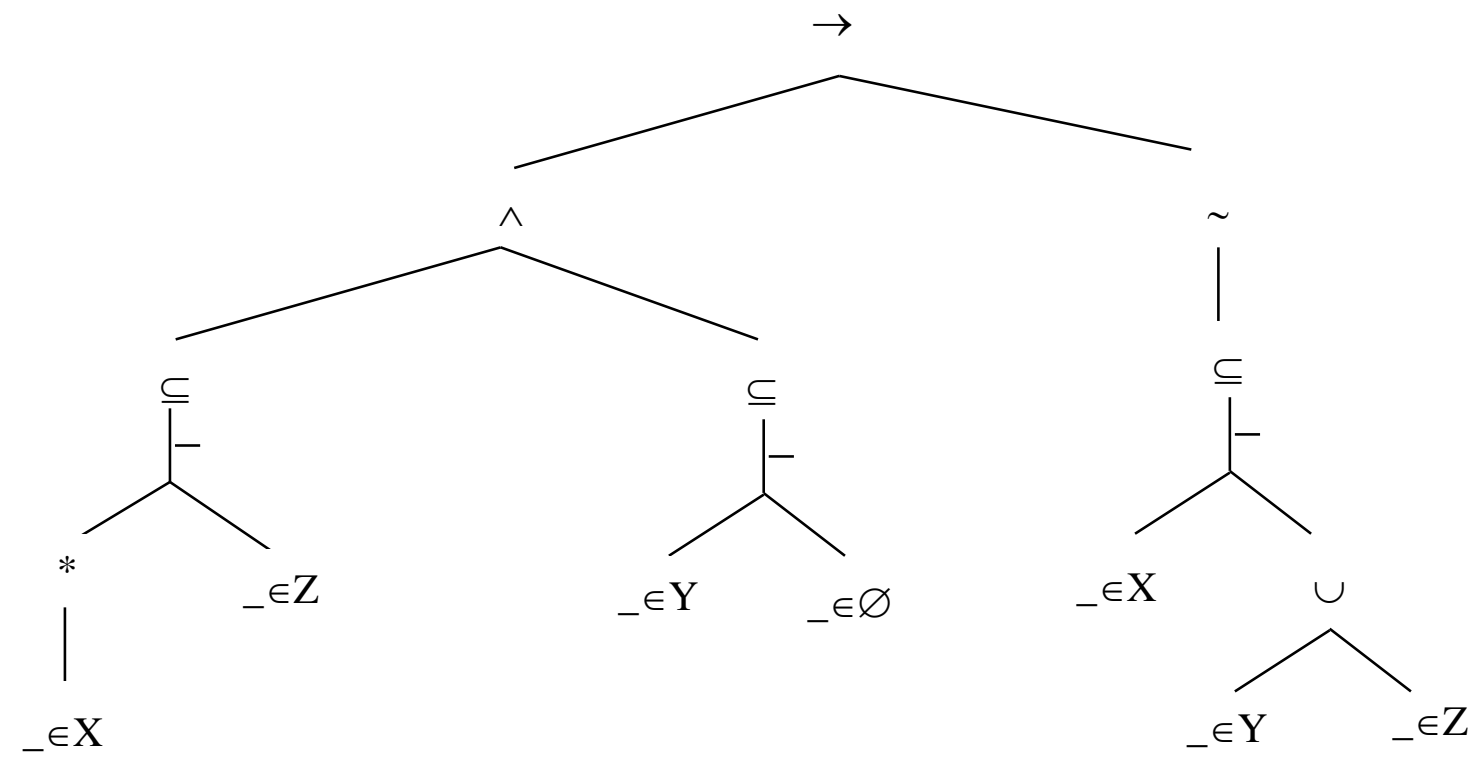

Figura 2. Árbol de la fórmula $\left(X^{*} \subseteq Z \wedge Y \subseteq \varnothing\right) \rightarrow \sim(X \subseteq Y \cup Z)$.

Para una fórmula $\alpha, \mathrm{H}(\alpha)$ el conjunto de hojas del $\operatorname{Ar}[\alpha]$, y $N(\alpha)$ el conjunto de nodos de $\operatorname{Ar}[\alpha]$.

Para cada fórmula a, una función de marca de hojas $m$ (o simplemente función de marca), es una función de $H(\alpha)$ en $\{0,1\}$.

Si $m(p)=1$ entonces se dice que la hoja $p$ está marcada con 1, o que es aceptada.

Si $m(p)=0$ entonces se dice que la hoja $p$ está marcada con 0 , o que es rechazada.

Cada función de marca de hojas $m$, se extiende a una función de marca de nodos, $M$, de $N(\alpha)$ en $\{0,1\}$, haciendo $M(h)=m(h)$ si h es una hoja, y aplicando las siguientes reglas primitivas para el forzamiento de marcas (las reglas primitivas para los operadores $\wedge, \vee, \rightarrow, \leftrightarrow \mathrm{y} \sim$ son las presentadas en Sierra (2006)):

\section{Reglas de instanciación o llenado de espacios vacíos}

Los espacios vacíos del árbol inicial deben ser instanciados, es decir, deben ser llenados con constantes o con variables. Cuando un espacio vacío se instancia con una constante o con una variable, todos los espacios vacíos asociados a este, se instancian con la misma constante o variable.

$\mathrm{IA} \subseteq$. Instanciación en la Aceptación de la Inclusión: Si una inclusión es aceptada, entonces los espacios vacíos asociados a esta inclusión son instanciados para toda constante ya dada y para toda variable ya dada.

$\mathrm{IR} \subseteq$. Instanciación en el Rechazo de la Inclusión: Si una inclusión es rechazada, entonces los espacios vacíos asociados a esta inclusión son instanciados para alguna constante nueva, es decir, una constante que no figura previamente en el árbol que se está marcando.

IC.

Instanciación en la Inclusión sin Marca: Si una inclusión no está marcada, entonces los espacios vacíos asociados a esta inclusión, pueden ser instanciados para alguna constante o variable.

Reglas para marcar los nodos cuyos espacios vacíos ya han sido instanciados

$\mathrm{R} \cup$. Rechazo de la Unión: Si una unión es rechazada, entonces tanto el hijo izquierdo como el derecho son rechazados.

$$
M(\cup)=0 \Rightarrow[M(i \cup)=0 \text { y } M(d \cup)=0]
$$

RiRd $\cup$. Rechazo a la Izquierda y Rechazo a la Derecha en la Unión: Si en una unión tanto el hijo izquierdo como el derecho son rechazados, entonces la unión es rechazada.

$$
[M(i \cup)=0 \text { y } M(d \cup)=0] \Rightarrow M(\cup)=0
$$

$\mathrm{R} \subseteq$. Rechazo de la Inclusión: Si una inclusión es rechazada, entonces el hijo izquierdo es aceptado y el hijo derecho es rechazado (para la constante nueva previamente instanciada en el espacio vacío).

$$
M(\subseteq)=0 \Rightarrow[M(i \subseteq)=1 \text { y } M(d \subseteq)=0]
$$

AiRd $\subseteq$. Aceptación a la Izquierda y Rechazo a la Derecha en la Inclusión: Si en una inclusión el hijo izquierdo es aceptado y el hijo derecho es rechazado, entonces la inclusión es rechazada. 
$[\mathrm{M}(\mathrm{i} \subseteq)=1$ y $\mathrm{M}(\mathrm{d} \subseteq)=0] \Rightarrow \mathrm{M}(\subseteq)=0$

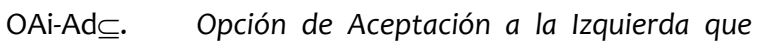
genera Aceptación a la Derecha en una Inclusión: Si se supone que para una variable independiente en el espacio vacío, el hijo izquierdo de una inclusión está marcado con 1 (paso inicial o supuesto) y al aplicar las reglas para marcar nodos, se tiene como consecuencia que el hijo derecho esté marcado con 1 (paso final), entonces la inclusión realmente está marcada con 1.

$$
[M(i \subseteq)=1 \Rightarrow M(d \subseteq)=1] \Rightarrow M(\subseteq)=1
$$

Los pasos que se encuentran entre el supuesto y el paso final (incluidos estos dos) se dice que están en el alcance del supuesto. Al aplicar la regla $\mathrm{OAi}-\mathrm{Ad} \subseteq$, resulta que $\mathrm{M}(\subseteq)=1 \mathrm{y}$ se dice que el supuesto ha sido descargado. Cuando esto ocurre, las marcas de los pasos que están en el alcance del supuesto, no pueden ser utilizadas en los pasos posteriores.

Una variable no es independiente en un paso dado, si la variable figura en un supuesto (la marca asignada no es el resultado de aplicar las reglas), y el paso dado se encuentra en el alcance de este supuesto.

A*. Aceptación del Complemento: Si un complemento es aceptado, entonces su alcance es rechazado.

$$
M(*)=1 \Rightarrow M\left(a^{*}\right)=0
$$

Ra*. Rechazo del Alcance del Complemento: $\mathrm{Si}$ el alcance de un complemento es rechazado, entonces el complemento es aceptado.

$M\left(a^{*}\right)=0 \Rightarrow M(*)=1$

RØ. Rechazo del Vacío: La hoja asociada al conjunto vacío es rechazada.

$\mathrm{M}(\mathrm{z} \in \varnothing)=0$, con $\mathrm{z}$ variable o constante.

AU. Aceptación del Universo de referencia: La hoja asociada al conjunto universal de referencia es aceptada.

$M(z \in U)=1$, con $z$ variable o constante.

\section{Validez de una fórmula}

Se dice que una fórmula a es A-válida (válida desde el punto de vista de los árboles) si y solamente si para toda función de marca $m$, se tiene que $M(R[a])=1$.

Se dice que una fórmula a es A-inválida si no es A-válida, es decir si existe una función de marca $m$, tal que $M(R[\alpha])=$ o. En este caso se dice que la función de marca refuta la fórmula $\alpha$. También se dice que el árbol de $\alpha$ está bien marcado ( $A B M$, todos sus nodos están marcados de acuerdo a las reglas sin generar contradicciones).

\section{REGLAS DERIVADAS PARA EL FORZAMIENTO DE MARCAS |}

Las reglas primitivas para el forzamiento de marcas, son suficientes para estudiar las propiedades de los árboles de forzamiento, pero en la práctica, cuando se trata de marcar todos los nodos de un árbol, es importante tener reglas que cubran todas las posibilidades. A continuación, se presenta un juego completo de reglas derivadas (las reglas derivadas para los conectivos $\wedge, \vee, \rightarrow, \leftrightarrow$ y $\sim$ se encuentran presentadas por Sierra (2006)).

Proposición 1. Reglas derivadas para la inclusión

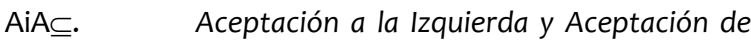
la Inclusión: Si son aceptados tanto la inclusión como su hijo izquierdo, entonces es aceptado el hijo derecho.

$[\mathrm{M}(\mathrm{i} \subseteq)=1$ y $\mathrm{M}(\subseteq)=1] \Rightarrow \mathrm{M}(\mathrm{d} \subseteq)=1$

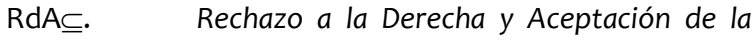
Inclusión: Si la inclusión es aceptada y su hijo derecho es rechazado, entonces es rechazado el hijo izquierdo.

$$
[M(d \subseteq)=0 \text { y } M(\subseteq)=1] \Rightarrow M(i \subseteq)=0
$$

Adᄃ. $\quad$ Aceptación a la Derecha en la Inclusión: Si en la inclusión se acepta el hijo derecho para una variable independiente en el espacio vacío, entonces se acepta la inclusión.

$$
M(d \subseteq)=1 \Rightarrow M(\subseteq)=1
$$

Riᄃ. $\quad$ Rechazo a la Izquierda en la Inclusión: Si en la inclusión se rechaza el hijo izquierdo para una variable independiente en el espacio vacío, entonces se acepta la inclusión.

$$
M(\mathrm{i} \subseteq)=0 \Rightarrow M(\subseteq)=1
$$

Prueba de $A i A \subseteq$ : Sean $M(i \subseteq)=1$ y $M(\subseteq)=1$. Supóngase que $M(d \subseteq)=0$, entonces se tiene que $M(i \subseteq)=1$ y $M(d \subseteq)=0$, y por AiRd $\subseteq$ se infiere $M(\subseteq)=0$, lo cual no es el caso. Por lo tanto, forzosamente $M(d \subseteq)=1$.

Prueba de $R d A \subseteq$ : Sean $M(d \subseteq)=0$ y $M(\subseteq)=1$. Supóngase que $M(i \subseteq)=1$, entonces se tiene que $M(i \subseteq)=1$ y $M(d \subseteq)=0$, y por AiRd $\subseteq$ se infiere $M(\subseteq)=0$, lo cual no es el caso. Por lo tanto, forzosamente $M(i \subseteq)=0$.

Prueba de $A d \subseteq$ : Sea $M(d \subseteq)=1$ para una variable independiente en el espacio vacío. Como $M(d \subseteq)=1$, se infiere que $M(i \subseteq)=1 \Rightarrow M(d \subseteq)=1$, lo cual por OAi-Ad $\subseteq$ implica que $M(\subseteq)=1$.

Prueba de $\mathrm{Ri} \subseteq$ : Sea $\mathrm{M}(\mathrm{i} \subseteq)$ = o para una variable independiente en el espacio vacío. Como $M(i \subseteq)=0$, se infiere que $\mathrm{M}(\mathrm{d} \subseteq)=0 \Rightarrow \mathrm{M}(\mathrm{i} \subseteq)=0$, lo cual es lógicamente equivalente a $M(i \subseteq)=1 \Rightarrow M(d \subseteq)=1$ y utilizando la regla OAiAd $\subseteq$ se concluye que $M(\subseteq)=1$. 
Proposición 2. Reglas derivadas para la unión

RiA $\cup$. Rechazo a la Izquierda y Aceptación de la Unión: Si se acepta una unión pero se rechaza su hijo izquierdo, entonces se acepta su hijo derecho.

$$
[M(i \cup)=0 \text { y } M(\cup)=1] \Rightarrow M(d \cup)=1
$$

RdA $\cup$ Rechazo a la Derecha $y$ Aceptación de la Unión: Si se acepta una unión pero se rechaza su hijo derecho, entonces se acepta su hijo izquierdo.

$$
[M(d \cup)=0 \text { y } M(\cup)=1] \Rightarrow M(i \cup)=1
$$

Aiv. Aceptación a la lzquierda en la Unión: Si se acepta el hijo izquierdo de una unión, entonces se acepta la unión.

$$
M(i \cup)=1 \Rightarrow M(\cup)=1
$$

Ad $\cup$.

Aceptación a la Derecha en la Unión: Si se acepta el hijo derecho de una unión, entonces se acepta la unión.

$$
M(d \cup)=1 \Rightarrow M(\cup)=1
$$

Prueba de RiA $\cup$ : Sean $M(i \cup)=0$ y $M(\cup)=1$. Supóngase que $M(d \cup)=0$, entonces se tiene que $M(i \cup)=M(d \cup)=0, y$ por RiRd $\cup$ se infiere $M(\cup)=0$, lo cual no es el caso. Por lo tanto, forzosamente $M(d \cup)=1$.

Prueba de RdA $\cup$ : Sean $M(d \cup)=0$ y $M(\cup)=1$. Supóngase que $M(i \cup)=0$, entonces se tiene que $M(i \cup)=M(d \cup)=0, y$ por RiRd $\cup$ se infiere $M(\cup)=0$, lo cual no es el caso. Por lo tanto, forzosamente $M(i \cup)=1$.

Prueba de $A i \cup$ : Sea $M(i \cup)=1$. Supóngase que $M(\cup)=0$, entonces por $R \cup$ se infiere $M(i \cup)=0$, lo cual no es el caso. Por lo tanto, forzosamente $M(\cup)=1$.

Prueba de $A d \cup$ : Sea $M(d \cup)=1$. Supóngase que $M(\cup)=$ 0 , entonces por $R \cup$ se infiere $M(d \cup)=0$, lo cual no es el caso. Por lo tanto, forzosamente $M(\cup)=1$.

Proposición 3. Reglas derivadas para el complemento

Aa*. Aceptación del Alcance del Complemento: Si el alcance de un complemento es aceptado, entonces el complemento es rechazado.

$M\left(a^{*}\right)=1 \Rightarrow M(*)=0$

$\mathrm{R}^{*}$. Rechazo del Complemento: Si un complemento es rechazado, entonces su alcance es aceptado.

$$
M(*)=0 \Rightarrow M\left(a^{*}\right)=1
$$

Prueba de $A a^{*}$ : Sea $M\left(a^{*}\right)=1$. Supóngase que $M(*)=1$, entonces por $A^{*}$ se infiere $M\left(a^{*}\right)=0$, lo cual no es el caso. Por lo tanto, forzosamente $M\left({ }^{*}\right)=0$.
Prueba de $R^{*}$ : Sea $M(*)=0$. Supóngase que $M\left(a^{*}\right)=0$, entonces por Ra* se infiere $M\left({ }^{*}\right)=1$, lo cual no es el caso. Por lo tanto, forzosamente $M\left(a^{*}\right)=1$.

\section{Proposición 4. Regla de opciones para la inclusión}

ORd-Ric. Opción de Rechazo a la Derecha que genera Rechazo a la Izquierda en una Inclusión: Si se supone que para una variable independiente en el espacio vacío, el hijo derecho de una inclusión está marcado con o (paso inicial o supuesto) y al aplicar las reglas para marcar nodos, se tiene como consecuencia que el hijo izquierdo esté marcado con o (paso final), entonces la inclusión realmente está marcado con 1.

$$
[M(d \subseteq)=0 \Rightarrow M(i \subseteq)=0] \Rightarrow M(\subseteq)=1
$$

Los pasos que se encuentran entre el supuesto y el paso final (incluidos estos dos) se dice que están en el alcance del supuesto. Al aplicar la regla ORd-Ri $\subseteq$, resulta que $M(\subseteq)=1$ y se dice que el supuesto ha sido descargado. Cuando esto ocurre, las marcas de los pasos que están en el alcance del supuesto, no pueden ser utilizadas en los pasos posteriores.

Una variable no es independiente en un paso dado, si la variable figura en un supuesto (la marca asignada no es el resultado de aplicar las reglas), y el paso dado se encuentra en el alcance de este supuesto.

Prueba de ORd-Riᄃ: Se tiene el supuesto inicial $M(d \subseteq)=$ $0 \Rightarrow M(i \subseteq)=0$. Si $M(i \subseteq)=1$, entonces por el supuesto inicial se infiere que $M(d \subseteq)=1$, resultando que, $M(i \subseteq)=1 \Rightarrow M(d \subseteq)$ $=1$, lo cual por la regla OAi-Ad $\subseteq$, implica que $M(\subseteq)=1$.

Proposición 5. Reglas de opciones para la unión

ORi-Ad $\cup$. Opción de Rechazo a la Izquierda que genera Aceptación a la Derecha en una Unión: Si se supone que el hijo izquierdo de una unión está marcado con $0, y$ al aplicar las reglas para marcar nodos se tiene como consecuencia que el hijo derecho esté marcado con 1, entonces la unión realmente está marcada con 1.

$$
[M(i \cup)=0 \Rightarrow M(d \cup)=1] \Rightarrow M(\cup)=1
$$

ORd-AiU. Opción de Rechazo a la Derecha que genera Aceptación a la Izquierda en una Unión: Si se supone que el hijo derecho de una unión está marcado con o, y al aplicar las reglas para marcar nodos se tiene como consecuencia que el hijo izquierdo esté marcado con 1, entonces la unión realmente está marcada con 1 .

$$
[M(d \cup)=0 \Rightarrow M(i \cup)=1] \Rightarrow M(\cup)=1
$$

Los pasos que se encuentran entre el supuesto y el paso final (incluidos estos dos) se dice que están en el alcance del supuesto. Al aplicar una de las reglas ORi-Ad $\cup$ o ORd-Ai $\cup$, resulta que $M(\cup)=1$ y se dice que el supuesto ha sido descargado. Cuando esto ocurre, las marcas de los pasos que están en el alcance del supuesto, no pueden ser utilizadas en los pasos posteriores. 
Prueba de ORi-Ad $\cup$ : Se tiene que $M(i \cup)=0 \Rightarrow M(d \cup)=$ 1. Supóngase que $M(\cup)=0$, entonces por $R \cup$ resulta que $M(i \cup)=M(d \cup)=0$, pero al tener $M(i \cup)=0$ se infiere que $M(d \cup)=1$, pero esto es imposible ya que se tiene que $M(d \cup)$ $=0$. Por lo tanto, forzosamente $M(\cup)=1$.

Prueba de ORd-Aiv: Se tiene que $M(d \cup)=0 \Rightarrow M(i \cup)=$ 1. Supóngase que $M(\cup)=0$, entonces por $R \cup$ resulta que $M(i \cup)=M(d \cup)=0$, pero al tener $M(d \cup)=0$ se infiere que $M(i \cup)=1$, pero esto es imposible ya que se tiene que $M(i \cup)=$ o. Por lo tanto, forzosamente $M(\cup)=1$.

\section{SEMÁNTICA DE VALORACIONES PARA LA LÓGICA DE CONJUNTOS |}

Una valoración $v$ es una función que interpreta las fórmulas atómicas de LC como elementos del conjunto $\{0,1\}$. Ampliando la presentación que hace Caicedo (1990) para el cálculo proposicional clásico, la valoración $v$ se extiende a una función $V$ que interpreta las fórmulas de LC en el conjunto $\{0,1\}$, mediante las siguientes reglas primitivas para las valoraciones, donde $z$ es una variable o una constante:

Vat. Si $W$ conjunto primitivo entonces $V(z \in W)=v(z \in W)$.

$V \sim . V(\sim A)=1 \Leftrightarrow V(A)=0$.

$\mathrm{V} \wedge . V(A \wedge B)=1 \Leftrightarrow V(A)=V(B)=1$.

$V \vee V(A \vee B)=0 \Leftrightarrow V(A)=V(B)=0$.

$\mathrm{V} \rightarrow \mathrm{V}(\mathrm{A} \rightarrow \mathrm{B})=0 \Leftrightarrow \mathrm{V}(\mathrm{A})=1$ y $\mathrm{V}(\mathrm{B})=0$.

$\mathrm{V} \leftrightarrow \mathrm{V}(\mathrm{A} \leftrightarrow \mathrm{B})=1 \Leftrightarrow \mathrm{V}(\mathrm{A})=\mathrm{V}(\mathrm{B})$

$\mathrm{V} \subseteq 1$. Si $\mathrm{V}(\mathrm{X} \subseteq \mathrm{Y})=0$ entonces existe una constante nueva, $a$, tal que $\mathrm{V}(a \in \mathrm{X})=1$ y $\mathrm{V}(a \in \mathrm{Y})=0$.

$\mathrm{V} \subseteq 2$. Si existe una constante o variable, $z$, tal que $V(z \in X)$ $=1 \mathrm{y} V(z \in \mathrm{Y})=0$ entonces $\mathrm{V}(\mathrm{X \subseteq Y})=0$.

$V \cup . V(z \in X \cup Y)=1 \Leftrightarrow(V(z \in X)=1 \circ V(z \in Y)=1)$.

$\mathrm{V}^{*} \cdot \mathrm{V}\left(\mathrm{z} \in \mathrm{X}^{*}\right)=1 \Leftrightarrow \mathrm{V}(\mathrm{z} \in \mathrm{X})=0$.

Se dice que una fórmula a es válida, si y solamente si para toda valoración $v, \mathrm{~V}(\alpha)=1$.

\section{EQUIVALENCIA DE LAS PRESENTACIONES PARA LA LÓGICA DE CONJUNTOS |}

Ampliando las definiciones de complejidad y de profundidad presentadas en Caicedo (1990) para el cálculo proposicional clásico, se define la Complejidad C, como una función la cual asigna a cada fórmula de LC un entero no negativo de la siguiente forma (donde $z$ es una variable o una constante):

$C(z \in W)=0$, donde $W$ es un conjunto primitivo. $\leftrightarrow\}$

$$
\begin{aligned}
& C(\sim \alpha)=1+C(\alpha) . \\
& C(z \in X \cup Y)=1+\text { máximo de }\{C(z \in X), C(z \in Y)\} \\
& C(X \subseteq Y)=1+\text { máximo de }\{C(z \in X), C(z \in Y)\} . \\
& C\left(z \in X^{*}\right)=1+C(z \in X) .
\end{aligned}
$$

Se define la Profundidad $\mathrm{P}$, como una función la cual asigna a cada nodo de un árbol un entero no negativo de la siguiente forma (la señal, $t$, puede ser una variable, una constante o el espacio vacío, ' _'):

$P(t \in W)=0$, donde $W$ es un conjunto primitivo.

$\mathrm{P}(\operatorname{Ar}[\alpha \mathrm{k} \beta])=1+$ máximo de $\{\mathrm{P}(\operatorname{Ar}[\alpha]), \mathrm{P}(\operatorname{Ar}[\beta])\}$, donde $\mathrm{k} \in\{\wedge, \vee, \rightarrow, \leftrightarrow\}$.

$$
\begin{aligned}
& \mathrm{P}(\operatorname{Ar}[\sim \alpha])=1+\mathrm{P}(\operatorname{Ar}[\alpha]) . \\
& \mathrm{P}(\operatorname{Ar}[\mathrm{X} \subseteq \mathrm{Y}])=1+\text { máximo de }\{\mathrm{P}(\operatorname{Ar}[t \in \mathrm{X}]), \mathrm{P}(\operatorname{Ar}[t \in \mathrm{Y}])\} . \\
& \mathrm{P}(\operatorname{Ar}[t \in \mathrm{X} \cup \mathrm{Y}])=1+\text { máximo de }\{\mathrm{P}(\operatorname{Ar}[t \in \mathrm{X}]), \mathrm{P}(\operatorname{Ar}[t \in \mathrm{Y}])\} . \\
& \mathrm{P}\left(\operatorname{Ar}\left[t \in \mathrm{X}^{*}\right]\right)=1+\mathrm{P}(\operatorname{Ar}[t \in \mathrm{X}]) .
\end{aligned}
$$

Teorema 1. Valoración asociada a una función de marca

Para cada fórmula a de LC y para cada función de marca $m$, existe una valoración $v_{m}$, tal que, $M(R[\alpha])=1 \Leftrightarrow V_{m}(\alpha)=1$.

Prueba: Sea a una fórmula de LC, y sea $m$ una función de marcas para $a$. Se define la función $v_{m}$ del conjunto de fórmulas atómicas en el conjunto $\{0,1\}$ de la siguiente forma:

Si $z \in W$ es una hoja (W es un conjunto primitivo, $z$ es una variable o una constante) entonces $v_{m}(z \in W)=m(z \in W)$.

La función $v_{m}$ se extiende a una función $V_{m}$ del conjunto de fórmulas de $L C$ en el conjunto $\{0,1\}$, mediante las reglas primitivas para las valoraciones de la sección: semántica de valoraciones para la lógica de conjuntos. Se tiene entonces que $V_{m}$ es una valoración de LC.

Para probar que $M(R[a])=1 \Leftrightarrow V_{m}(\alpha)=1$, se procede por inducción sobre la complejidad de la fórmula $a$.

Paso base: Supóngase que la $C(\alpha)=0$, esto significa que $a=z \in W$ donde $W$ es un conjunto primitivo y $z$ es una variable o una constante, y por lo tanto se tienen $R[\alpha]=\alpha$, $M(\alpha)=m(\alpha), v_{m}(\alpha)=m(\alpha)$ y $v_{m}(\alpha)=v_{m}(\alpha)$. Se concluye entonces que $M(R[a])=1 \Leftrightarrow V_{m}(\alpha)=1$.

Paso de Inducción: Supóngase que $C(\alpha) \geq 1$.

Al ser $C(\alpha) \geq 1$, $a$ debe ser una fórmula compuesta, es decir, a tiene una de las siguientes formas: $z \in X \cup Y, X \subseteq Y$, $z \in X^{*}$ (los casos en los cuales $a$ tiene una de las siguientes formas: $\beta \wedge \gamma, \beta \vee \gamma, \beta \rightarrow \gamma, \beta \leftrightarrow \gamma, \sim \beta$, se encuentran probados en Sierra (2006)). Se analiza cada caso por separado.

Caso 1: Sea $\alpha=z \in X \cup Y$. Se tiene que $R[\alpha]=\cup$, por lo que $M(R[a])=0 \Leftrightarrow M(\cup)=0$, pero por $R \cup$ se tiene $M(\cup)=0 \Leftrightarrow$ $[M(i \cup)=M(d \cup)=0]$, y como además $i \cup=R[z \in X]$ y $d \cup=$ 
$R[z \in Y]$, resulta que $M(R[\alpha])=0 \Leftrightarrow[M(R[z \in X])=M(R[z \in Y])$ $=0]$. Utilizando la hipótesis inductiva se tiene que $M(R[\alpha])=$ $0 \Leftrightarrow\left[V_{m}(z \in X)=V_{m}(z \in Y)=0\right]$. Por la regla primitiva $V \cup$ para la valoración $V_{m}$, se concluye que $M(R[\alpha])=0 \Leftrightarrow V_{m}(z \in X \cup Y)$ $=0$, es decir, $M(R[\alpha])=1 \Leftrightarrow V_{m}(\alpha)=1$.

Caso 2: Sea $\alpha=X \subseteq Y$, por lo que $R[\alpha]=\subseteq$. Si $M(R[\alpha])=0$ entonces $M(\subseteq)=0$, por las reglas $I R \subseteq y \quad R \subseteq$ se infiere $M(i \subseteq)=$ 1 y $\mathrm{M}(\mathrm{d} \subseteq)=0$ para alguna constante, $a$, nueva en el espacio vacío, y como además $\mathrm{i} \subseteq=\mathrm{R}[a \in \mathrm{X}]$ y $\mathrm{d} \subseteq=\mathrm{R}[a \in \mathrm{Y}]$, resulta que $M(R[a \in X])=1$ y $M(R[a \in Y])=0$. Utilizando la hipótesis inductiva resulta que $V_{m}(a \in \mathrm{X})=1$ y $V_{m}(a \in \mathrm{Y})=0$, y por la regla primitiva $V_{\subseteq 2}$ para la valoración $V_{m}$, se concluye que $V_{m}(X \subseteq Y)=0$. Se ha probado de esta manera que $M(R[\alpha])=0$ $\Rightarrow V_{m}(X \subseteq Y)=0$.

Si $V_{m}(\mathrm{X} \subseteq \mathrm{Y})=0$, por la regla primitiva $\mathrm{V}_{\subseteq 1}$ para la valoración $V_{m}$, resulta $V_{m}(a \in X)=1$ y $V_{m}(a \in Y)=0$ para alguna constante, $a$, nueva. Utilizando la hipótesis inductiva se infiere $M(R[a \in X])=1$ y $M(R[a \in Y])=0$, es decir, $M(i \subseteq)=1 \mathrm{y}$ $M(d \subseteq)=0$, aplicando la regla AiRd $\subseteq$ se obtiene $M(\subseteq)=0$, es decir, $M(R[a])=0$. Se ha probado que $V_{m}(X \subseteq Y)=0 \Rightarrow$ $M(R[a])=0$, y como ya se probó la recíproca, se obtiene $M(R[\alpha])=0 \Leftrightarrow V_{m}(X \subseteq Y)=0$, y por lo tanto, $M(R[\alpha])=1 \Leftrightarrow$ $V_{m}(a)=1$.

Caso 3: Sea $a=z \in X^{*}$. Se tiene que $R[\alpha]=*$, por lo que $M(R[a])=1 \Leftrightarrow M(*)=1$, pero por $A^{*}$ se tiene $M\left(^{*}\right)=1 \Leftrightarrow$ $M\left(a^{*}\right)=0, y$ como además $a^{*}=R[z \in X]$, resulta que $M(R[a])$ $=1 \Leftrightarrow[M(R[z \in X])=0]$. Utilizando la hipótesis inductiva se tiene que $M(R[\alpha])=1 \Leftrightarrow V_{m}(z \in X)=0$. Por la regla primitiva $V^{*}$ para la valoración $V_{m}$, se concluye que $M(R[\alpha])=1 \Leftrightarrow$ $V_{m}\left(z \in X^{*}\right)=1$, es decir, $M(R[\alpha])=1 \Leftrightarrow V_{m}(\alpha)=1$.

Se tiene entonces que para todos los casos $M(R[a])=1$ $\Leftrightarrow V_{m}(\alpha)=1$, quedando así probado el paso de inducción. Por el principio de Inducción se concluye que: $M(R[\alpha])=1 \Leftrightarrow$ $V_{m}(\alpha)=1$.

Se ha probado entonces que para cada fórmula a de LC y para cada función de marca $m$, existe una valoración $v_{m}$, tal que, $M(R[\alpha])=1 \Leftrightarrow V_{m}(\alpha)=1$.

\section{Teorema 2. Función de marca asociada a una valoración}

Para cada fórmula a de LC y para cada valoración $v$, existe una función de marca $m_{v}$, tal que, $M_{v}(R[a])=1 \Leftrightarrow V(\alpha)$ $=1$.

Prueba: Sea a una fórmula de LC y sea $v$ una valoración. Se define la función de marca $m_{v}$ del conjunto de hojas del árbol de $a$ en el conjunto $\{0,1\}$ de la siguiente forma:

Si $\mathrm{W}$ es un conjunto primitivo y $z$ es una variable o una constante entonces $m_{v}(z \in W)=v(z \in W)$

La función $m_{v}$ se extiende a una función $M v$ del conjunto de nodos del árbol de $\alpha$ en el conjunto $\{0,1\}$, mediante las reglas primitivas para el forzamiento de marcas presentadas en la sección: marcando los nodos de un árbol. Se tiene entonces que $M_{v}$ es una función de marca de nodos.

Para probar que $M_{v}(R[\alpha])=1 \Leftrightarrow V(\alpha)=1$, se procede por inducción sobre la profundidad de $\operatorname{Ar}[\alpha]$.

Paso base: Supóngase que $P(\operatorname{Ar}[\alpha])=0$, esto significa que $\alpha=z \in W$ donde $W$ es un conjunto primitivo y $z$ es una variable o una constante, y por lo tanto se tienen $\mathrm{R}[\alpha]=\alpha$, $M_{v}(\alpha)=m_{v}(\alpha), m_{v}(\alpha)=v(\alpha)$ y $v(\alpha)=V(\alpha)$. Se concluye entonces que $M_{v}(R[a])=1 \Leftrightarrow V(\alpha)=1$.

Paso de inducción: Supóngase que $\operatorname{P}(\operatorname{Ar}[\alpha]) \geq 1$.

Al ser $P(\operatorname{Ar}[\alpha]) \geq 1$, a debe ser una fórmula compuesta, es decir, a tiene una de las siguientes formas: $z \in X \cup Y, X \subseteq Y$, $z \in X^{*}$ (los casos en los cuales $\alpha$ tiene una de las siguientes formas: $\beta \wedge \gamma, \beta \vee \gamma, \beta \rightarrow \gamma, \beta \leftrightarrow \gamma, \sim \beta$, se encuentran probados en Sierra (2006)). Se analiza cada caso por separado.

Caso 1: Sea $\alpha=z \in X \cup Y$. Se tiene que $R[\alpha]=\cup$, por lo que $M_{v}(R[a])=0 \Leftrightarrow M_{v}(\cup)=0$, pero por la regla primitiva $R \cup$ para la función de marca $M_{v}$, se tiene $M_{v}(\cup)=0 \Leftrightarrow\left[M_{v}(i \cup)=\right.$ $\left.M_{v}(\mathrm{~d} \cup)=0\right]$, y como además $i \cup=R[z \in X]$ y $d \cup=R[z \in Y]$, resulta que $M_{v}(R[\alpha])=0 \Leftrightarrow\left[M_{v}(R[z \in X])=M_{v}(R[z \in Y])=0\right]$. Utilizando la hipótesis inductiva se tiene que $M_{v}(R[\alpha])=0 \Leftrightarrow$ $[\mathrm{V}(z \in \mathrm{X})=\mathrm{V}(z \in \mathrm{Y})=0]$. Por la definición de $\mathrm{V} \cup$ se concluye que $M_{v}(R[a])=0 \Leftrightarrow V(z \in X \cup Y)=0$, es decir, $M_{v}(R[a])=1 \Leftrightarrow$ $\mathrm{V}(\alpha)=1$.

Caso 2: Sea $\alpha=\mathrm{X} \subseteq \mathrm{Y}$, por lo que $\mathrm{R}[\alpha]=\subseteq$. Si $M_{v}(\mathrm{R}[\alpha])=0$ entonces $M_{v}(\subseteq)=0$, por las reglas primitivas $I R \subseteq y \quad R \subseteq$ para la función de marca $M_{v}$, se obtiene $M_{v}(\mathrm{i} \subseteq)=1$ y $M_{v}(\mathrm{~d} \subseteq)$ = o para alguna constante, $a$, nueva en el espacio vacío, y como además $\mathrm{i} \subseteq=\mathrm{R}[a \in \mathrm{X}]$ y $\mathrm{d} \subseteq=\mathrm{R}[a \in \mathrm{Y}]$, resulta que $M_{v}(\mathrm{R}[a \in \mathrm{X}])=$ 1 y $M_{v}(R[a \in \mathrm{Y}])=0$. Utilizando la hipótesis inductiva se infiere $\mathrm{V}(a \in \mathrm{X})=1$ y $\mathrm{V}(a \in \mathrm{Y})=0$. Por la definición de $\mathrm{V} \subseteq 2$ se concluye que $\mathrm{V}(\mathrm{X} \subseteq \mathrm{Y})=0$, es decir, $\mathrm{V}(\alpha)=0$. Se ha probado que $M_{v}(R[\alpha])=0 \Rightarrow V(\alpha)=0$.

Si $\mathrm{V}(\alpha)=0$, resulta que $\mathrm{V}(\mathrm{X} \subseteq \mathrm{Y})=0$, lo cual por $\mathrm{V} \subseteq 1$ significa $\mathrm{V}(a \in \mathrm{X})=1$ y $\mathrm{V}(a \in \mathrm{Y})=0$ para alguna constante, $a$, nueva. Utilizando la hipótesis inductiva se infiere $M_{v}(R[a \in X])$ $=1$ y $M_{v}(\mathrm{R}[a \in \mathrm{Y}])=0$, es decir, $M_{v}(\mathrm{i} \subseteq)=1$ y $M_{v}(\mathrm{~d} \subseteq)=0$, finalmente aplicando la regla primitiva AiRd $\subseteq$ para la función de marca $M_{v}$, se infiere que $M_{v}(\subseteq)=0$, es decir, $M_{v}(R[\alpha])=0$. Se ha probado que $V(\alpha)=0 \Rightarrow M_{v}(R[\alpha])=0$, y como ya se probó la recíproca, se concluye $V(\alpha)=0 \Leftrightarrow M_{v}(R[\alpha])=0$, es decir, $M_{v}(R[a])=1 \Leftrightarrow V(\alpha)=1$.

Caso 3: Sea $\alpha=z \in X^{*}$. Se tiene que $R[\alpha]=*$, por lo que $M_{v}(R[\alpha])=1 \Leftrightarrow M_{v}(*)=1$, pero por la regla primitiva $A^{*}$ para la función de marca $M_{v}$, se tiene $M_{v}(*)=1 \Leftrightarrow M_{v}\left(a^{*}\right)=0$, y como además $a^{*}=\mathrm{R}[z \in \mathrm{X}]$, resulta que $M_{v}(\mathrm{R}[\alpha])=1 \Leftrightarrow$ $M_{v}(R[z \in X])=0$. Utilizando la hipótesis inductiva se tiene que $M_{V}(R[\alpha])=1 \Leftrightarrow V(z \in X)=0$. Por la definición de $V^{*}$ se concluye que $M_{v}(R[\alpha])=1 \Leftrightarrow V\left(z \in X^{*}\right)=1$, es decir, $M_{v}(R[a])$ $=1 \Leftrightarrow \mathrm{V}(\alpha)=1$. 
Se tiene entonces que para todos los casos $M_{v}(R[\alpha])=1$ $\Leftrightarrow V(\alpha)=1$, quedando así probado el paso de inducción. Por el principio de Inducción se concluye que: $M_{v}(R[\alpha])=1 \Leftrightarrow$ $\mathrm{V}(\alpha)=1$.

Se ha probado entonces que para cada fórmula a de LC y para cada valoración $v$, existe una función de marca $m_{v}$, tal que, $M_{v}(R[\alpha])=1 \Leftrightarrow V(\alpha)=1$.

Teorema 3. Caracterización semántica y deductiva de los árboles de forzamiento

La fórmula a es válida desde el punto de vista de los árboles si y solamente si a es válida desde el punto de vista de las valoraciones.

Prueba: Supóngase que a no es válida desde el punto de vista de los árboles, entonces existe una función de marca $m$, tal que $M(R[\alpha])=0$. Se tiene entonces, por la Teorema 1, que existe una valoración $v_{m}$, tal que $V_{m}(\alpha)=0$, y por lo tanto, $\alpha$ no puede ser válida desde el punto de vista de las valoraciones.

Supóngase ahora que a no es válida desde el punto de vista de las valoraciones, entonces existe una valoración $v$, tal que $V(\alpha)=0$. Se tiene entonces, por la Teorema 2, que existe una función de marca $m_{v}$, tal que $M_{v}(R[\alpha])=0$, y por lo tanto, a no puede ser válida desde el punto de vista de los árboles.

Se concluye así que a es válida desde el punto de vista de los árboles si y solamente si a es válida desde el punto de vista de las valoraciones.

\section{OTRAS OPERACIONES ENTRE CONJUNTOS}

Siguiendo a Oubiña (1965), las operaciones de intersección, diferencia y diferencia simétrica entre conjuntos, así como la relación de igualdad entre los mismos, se definen de la siguiente manera: $A \cong B$ significa que $(A \subseteq B) \wedge(B \subseteq A)$, $A \cap B=\left(A^{*} \cup B^{*}\right) *, A / B=A \cap B^{*}, A \triangle B=(A \cup B) /(A \cap B)$. Los árboles de estas operaciones entre conjuntos se construyen de manera similar a los árboles de la unión de conjuntos, el árbol de la relación de igualdad de conjuntos se construye de manera similar al árbol de la inclusión de conjuntos.

Como consecuencia de estas definiciones, de las reglas primitivas y de las proposiciones 1 a 5 , se tiene el siguiente juego completo de reglas para el forzamiento de marcas:

Reglas de instanciación o llenado de espacios vacíos

$\mathrm{IA} \cong$. Instanciación en la Aceptación de la lgualdad: Si una igualdad es aceptada, entonces los espacios vacíos asociados a esta igualdad son instanciados para toda constante ya dada y para toda variable ya dada.

$\mathrm{IR} \cong . \quad$ Instanciación en el Rechazo de la lgualdad: Si una igualdad es rechazada, entonces los espacios vacíos asociados a esta igualdad son instanciados para alguna constante nueva.
I․ Instanciación en la lgualdad sin Marca: Si una igualdad no está marcada, entonces los espacios vacíos asociados a esta igualdad, pueden ser instanciados para alguna constante o variable.

$$
\begin{array}{ll}
R \cong . & \text { Rechazo de la lgualdad: } \\
& M(\cong)=0 \Rightarrow M(i \cong) \neq M(d \cong)
\end{array}
$$

Reglas para marcar los nodos cuyos espacios vacíos ya han sido instanciados

$\mathrm{Mi} \neq \mathrm{Md \cong .} \mathrm{Diferentes} \mathrm{Marcas} \mathrm{a} \mathrm{la} \mathrm{Izquierda} \mathrm{y} \mathrm{a} \mathrm{la} \mathrm{Derecha}$ en la lgualdad:

$$
M(i \cong) \neq M(d \cong) \Rightarrow M(\cong)=0
$$

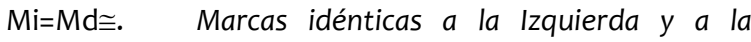
Derecha en la lgualdad (para una variable independiente en el espacio vacío):

$$
\mathrm{M}(\mathrm{i} \cong)=\mathrm{M}(\mathrm{d} \cong) \Rightarrow \mathrm{M}(\cong)=1
$$

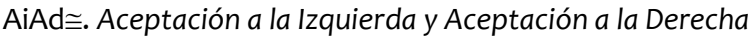
en la lgualdad (para una variable independiente en el espacio vacío):

$$
[M(i \cong)=1 \text { y } M(d \cong)=1] \Rightarrow M(\cong)=1
$$

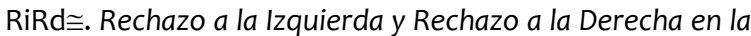
lgualdad (para una variable independiente en el espacio vacío):

$$
[M(i \cong)=0 \text { y } M(d \cong)=0] \Rightarrow M(\cong)=1
$$

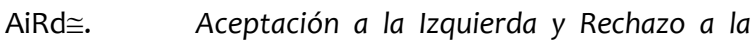
Derecha en la lgualdad:

$$
[M(\mathrm{i} \cong)=1 \text { y } M(d \cong)=0] \Rightarrow M(\cong)=0
$$

$\mathrm{AiA} \cong$. Aceptación a la Izquierda y Aceptación de la Igualdad:

$$
[M(i \cong)=1 \text { y } M(\cong)=1] \Rightarrow M(d \cong)=1
$$

$\mathrm{RdA} \cong$. Rechazo a la Derecha y Aceptación de la lgualdad:

$$
[\mathrm{M}(\mathrm{d} \cong)=0 \text { y } \mathrm{M}(\cong)=1] \Rightarrow M(\mathrm{i} \cong)=0
$$

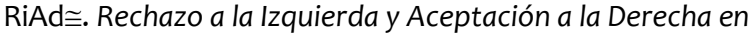
la lgualdad:

$[M(i \cong)=0$ y $M(d \cong)=1] \Rightarrow M(\cong)=0$

$\mathrm{RiA} \cong$. Rechazo a la Izquierda y Aceptación de la lgualdad:

$$
[M(i \cong)=0 \text { y } M(\cong)=1] \Rightarrow M(d \cong)=0
$$

$\mathrm{AdA} \cong$. Aceptación a la derecha y Aceptación de la Igualdad:

$$
[M(d \cong)=1 \text { y } M(\cong)=1] \Rightarrow M(i \cong)=1
$$

$\mathrm{A} \cap$. Aceptación de la Intersección:

$$
M(\cap)=1 \Rightarrow[M(\mathrm{i} \cap)=1 \text { y } M(d \cap)=1]
$$


AiAd $\cap$. Aceptación a la Izquierda y Aceptación a la Derecha en la Intersección:

$$
[\mathrm{M}(\mathrm{i} \cap)=1 \text { y } \mathrm{M}(\mathrm{d} \cap)=1] \Rightarrow \mathrm{M}(\cap)=1
$$

AiR $\cap$. Aceptación a la Izquierda y Rechazo de la Intersección:

$[M(\mathrm{i} \cap)=1$ y $M(\cap)=0] \Rightarrow M(d \cap)=0$

AdR $\cap$. Aceptación a la Derecha y Rechazo de la Intersección:

$[M(d \cap)=1$ y $M(\cap)=0] \Rightarrow M(i \cap)=0$

Rin. Rechazo a la Izquierda en la Intersección:

$M(\mathrm{i} \cap)=0 \Rightarrow M(\cap)=0$

$\mathrm{Rd} \cap$. Rechazo a la Derecha en la Intersección:

$M(\mathrm{~d} \cap)=0 \Rightarrow M(\cap)=0$

$\mathrm{A} \triangle$. Aceptación de la Diferencia simétrica:

$\mathrm{M}(\Delta)=1 \Leftrightarrow M(\mathrm{i} \Delta) \neq \mathrm{M}(\mathrm{d} \Delta)$

AiAd $\Delta$. Aceptación a la Izquierda y Aceptación a la Derecha en la Diferencia simétrica:

$[\mathrm{M}(\mathrm{i} \Delta)=1$ y $\mathrm{M}(\mathrm{d} \Delta)=1] \Rightarrow \mathrm{M}(\Delta)=0$

$\operatorname{RiRd} \Delta$. Rechazo a la Izquierda y Rechazo a la Derecha en la Diferencia simétrica:

$[M(\mathrm{i} \Delta)=0$ y $M(\mathrm{~d} \Delta)=0] \Rightarrow M(\Delta)=0$

AiRd $\Delta$. Aceptación a la Izquierda y Rechazo a la Derecha en la Diferencia simétrica:

$[M(\mathrm{i} \Delta)=1$ y $M(d \Delta)=0] \Rightarrow M(\Delta)=1$

AiA $\triangle$. Aceptación a la Izquierda y Aceptación de la Diferencia simétrica:

$[\mathrm{M}(\mathrm{i} \Delta)=1$ y $\mathrm{M}(\Delta)=1] \Rightarrow M(\mathrm{~d} \Delta)=0$

$\operatorname{RdA} \Delta$. Rechazo a la Derecha y Aceptación de la Diferencia simétrica:

$[\mathrm{M}(\mathrm{d} \Delta)=0$ y $\mathrm{M}(\Delta)=1] \Rightarrow \mathrm{M}(\mathrm{i} \Delta)=1$

$\operatorname{RiAd} \Delta$. Rechazo a la Izquierda y Aceptación a la Derecha en la Diferencia simétrica:

$[M(\mathrm{i} \Delta)=0$ y $M(d \Delta)=1] \Rightarrow M(\Delta)=1$

$\mathrm{RiA} \triangle$. Rechazo a la Izquierda y Aceptación de la Diferencia simétrica:

$[M(\mathrm{i} \Delta)=0$ y $M(\Delta)=1] \Rightarrow M(d \Delta)=1$

$\mathrm{AdA} \Delta$. Aceptación a la derecha y Aceptación de la Diferencia simétrica:

$[M(d \Delta)=1$ y $M(\Delta)=1] \Rightarrow M(i \Delta)=0$
RiR $\Delta$. Rechazo a la Izquierda y Rechazo de la Diferencia simétrica:

$$
[\mathrm{M}(\mathrm{i} \Delta)=0 \text { y } \mathrm{M}(\Delta)=0] \Rightarrow \mathrm{M}(\mathrm{d} \Delta)=0
$$

AiR $\Delta$. Aceptación a la Izquierda y Rechazo de la Diferencia simétrica:

$$
[\mathrm{M}(\mathrm{i} \Delta)=1 \text { y } \mathrm{M}(\Delta)=0] \Rightarrow \mathrm{M}(\mathrm{d} \Delta)=1
$$

$\operatorname{RdR} \Delta$. Rechazo a la Derecha y Rechazo de la Diferencia simétrica:

$[\mathrm{M}(\mathrm{d} \Delta)=0$ y $\mathrm{M}(\Delta)=0] \Rightarrow \mathrm{M}(\mathrm{i} \Delta)=0$

AdR $\Delta$. Aceptación a la Derecha y Rechazo de la Diferencia simétrica:

$[\mathrm{M}(\mathrm{d} \Delta)=1$ y $\mathrm{M}(\Delta)=0] \Rightarrow \mathrm{M}(\mathrm{i} \Delta)=1$

A/. Aceptación de la Diferencia:

$M(/)=1 \Rightarrow[M(\mathrm{i} /)=1$ y $M(\mathrm{~d} /)=0]$

AiRd/. Aceptación a la Izquierda y Rechazo a la Derecha en la Diferencia:

$[M(\mathrm{i} /)=1$ y $M(\mathrm{~d} /)=0] \Rightarrow M(/)=1$

AiR/. Afirmación a la Izquierda y Rechazo de la Diferencia:

$[\mathrm{M}(\mathrm{i} /)=1$ y $\mathrm{M}(/)=0] \Rightarrow \mathrm{M}(\mathrm{d} /)=1$

RdR/. Rechazo a la Derecha y Rechazo de la Diferencia:

$[M(d /)=0$ y $M(/)=0] \Rightarrow M(\mathrm{i} /)=0$

Ri/. Rechazo a la Izquierda en la Diferencia:

$M(\mathrm{i} /)=0 \Rightarrow M(/)=0$

Ad/. Afirmación a la Derecha en la Diferencia:

$M(d /)=1 \Rightarrow M(/)=0$

\section{ILUSTRACIONES}

Siguiendo a (Sierra, 2001; Sierra, 2006), un árbol de forzamiento está mal marcado, cuando su raíz está marcada con 0 , y además existen dos nodos asociados a una misma fórmula, los cuales tienen marcas contrarias. Lo anterior significa que cuando el árbol de una fórmula está mal marcado, entonces la fórmula es A-válida. En la Fig. 3 se muestra un árbol de forzamiento mal marcado para la fórmula $A$-válida $A \Delta U^{*} \cong B \cup \phi \rightarrow A \subseteq B$. Un nodo encerrado en un círculo indica que el nodo está marcado con 1 , un nodo encerrado en un cuadro indica que el nodo está marcado con 0 .

Justificaciones:
1. RR.
2, 3. $\mathrm{R} \rightarrow$ en 1

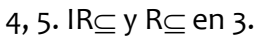
6. IA $\cong$ en 2 y IA en 4 .
7. AU.
8. Aa* en 7 . 


\section{9. $A i R d \Delta$ en 8 y $6 . \quad$ 10. $A i A \cong$ en 9 y $2 . \quad$ 11. $R \phi$.}

12. RdA $\cup$ en 11 y $10 . \quad$ 13. DM en 5 y 12.

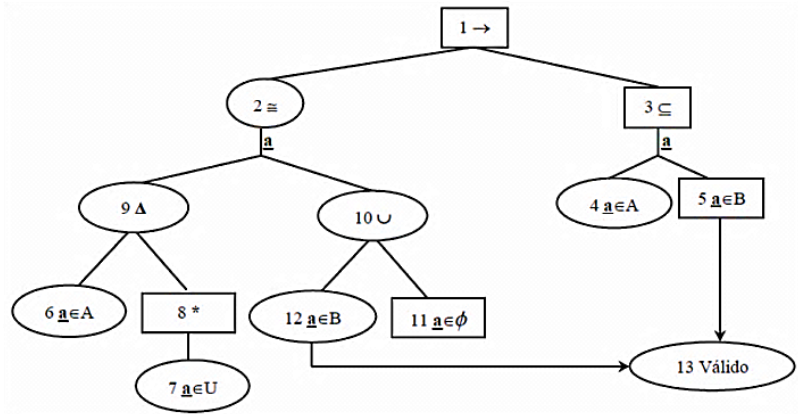

Figura 3. Árbol de forzamiento para la fórmula $A \triangle U^{*} \cong B \cup \phi \rightarrow A \subseteq B$.

En el siguiente ejemplo aplicativo, se ilustra la construcción de un contraejemplo, con el cual se refuta la validez de un razonamiento dado.

En cierta institución educativa se realizaron 2 reuniones. A la primera reunión asistieron todos los estudiantes que matricularon artes junto con todos los estudiantes que matricularon biología, a la segunda reunión asistieron todos los estudiantes que matricularon contabilidad junto con todos los estudiantes que matricularon artes. El organizador de las reuniones, observa que en ambas reuniones estuvieron presentes exactamente los mismos estudiantes, y en consecuencia, concluye que todos los estudiantes que matricularon biología también matricularon contabilidad. Es acertada la conclusión del organizador de las reuniones?

Si A representa el conjunto de estudiantes que matricularon artes, $B$ representa el conjunto de estudiantes que matricularon biología y $C$ representa el conjunto de estudiantes que matricularon contabilidad, entonces en el lenguaje de la lógica de conjuntos, el razonamiento del organizador de las reuniones queda representado por la fórmula $A \cup B \cong A \cup C \rightarrow B \subseteq C$, y la pregunta sobre la inferencia hecha por el mismo, se reduce al análisis de validez de esta fórmula.

En la Fig. 4 se muestra un árbol de forzamiento bien marcado para la fórmula $A$-inválida $A \cup B \cong A \cup C \rightarrow B \subseteq C$.

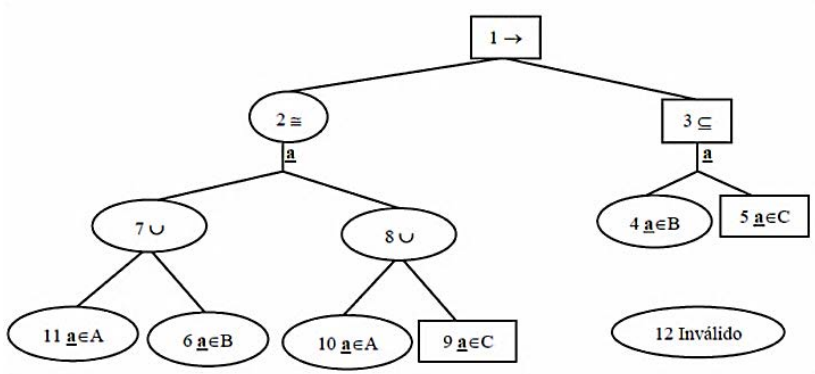

Figura 4. Árbol de forzamiento para la fórmula $A \cup B \cong A \cup C \rightarrow B \subseteq C$.
Justificaciones:
1. RR.
2, 3. $\mathrm{R} \rightarrow$ en 1 .

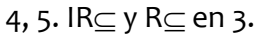

6. $I A \cong$ en 2 y $I A$ en 4 . 7. Ad $\cup$ en 6

8. $A i A \cong$ en 7 y 2 .

9. IR en 5 .

10. $R d A \cup 9$ y 8.

11. IA en 10.

12. ABM.

Observar que las marcas de los nodos asociados a las hojas determinan una valoración ( $y$ en consecuencia los conjuntos primitivos) que refuta a la fórmula analizada: $\mathrm{V}(\mathrm{a} \in \mathrm{A})=\mathrm{V}(\mathrm{a} \in \mathrm{B})=1$ y $\mathrm{V}(\mathrm{a} \in \mathrm{C})=0$, es decir, $\mathrm{A}=\mathrm{B}=\{\mathrm{a}\}, \mathrm{C}=\varnothing$. Se verifica que $A \cup B \cong A \cup C$ pero $\sim(B \subseteq C)$.

Regresando al ejemplo aplicativo, se tiene que la conclusión del organizador de reuniones no es acertada, puesto que, si hay un único estudiante que matriculó artes, y también él mismo, es el único que matriculó biología, pero además nadie matriculó contabilidad, entonces resulta, que en ambas reuniones estuvieron presentes los mismos estudiantes, pero claramente, no es cierto que todos los estudiantes que matricularon biología también matricularon contabilidad.

En la Fig. 5 se muestra un árbol de forzamiento con la raíz marcada con 1 para la fórmula $A$-válida $(A \subseteq B \wedge B \cap C \cong B) \rightarrow$ $A \cup C \subseteq C$.

\section{Justificaciones:}

1. OA.

2, 3. A^ en 1.

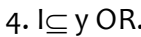

5. IA $\cong y$ IR en 4 .

6. $R d \cap$ en 5 .

7. $R i A \cong$ en 6 y 3 .

8. IA $\subseteq$ y IR en 7 .

9. $R d A \subseteq$ en 8 y 2 .

10. IR en 9.

11. IR en 4. 12. RiRd $\cup$ en 10 y 11. 13. ORd-Ri $\subseteq$ en 4 y 12.

14. OAi-Ad $\rightarrow$ en 1, 13. 15. Raíz marcada con 1.

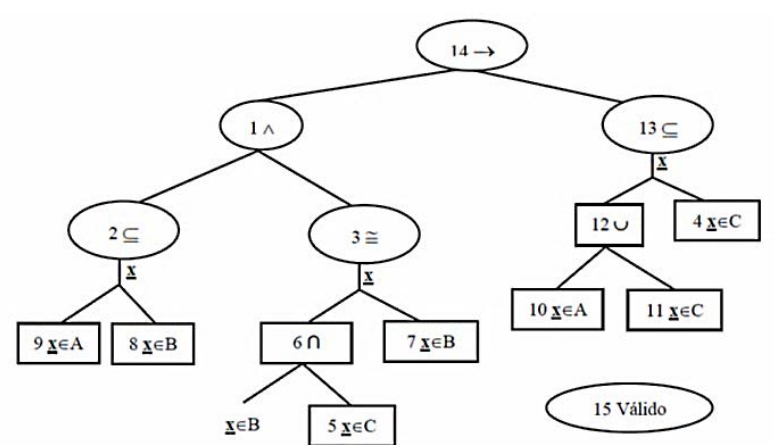

Figura 5. Árbol de forzamiento para la fórmula $(A \subseteq B \wedge B \cap C \cong B) \rightarrow$ $A \cup C \subseteq C$.

\section{CONCLUSIONES}

Con los árboles de forzamiento semántico para operaciones entre conjuntos es posible determinar la validez de una fórmula que involucre operaciones entre conjuntos, de manera visual y completamente mecánica, por ejemplo, 
recorriendo el árbol de la fórmula mediante un algoritmo y en cada nodo buscando la aplicación de una regla para marcarlos. Cuando el árbol de una fórmula está bien marcado, es decir, cuando la fórmula es inválida, entonces la lectura de las marcas de las fórmulas atómicas, proporciona una valoración que refuta la validez de la fórmula, y además, con esta valoración se construyen los conjuntos que la refutan, es decir, los conjuntos que generan el contraejemplo.

En Sierra (2001) se presentan los árboles de forzamiento para la lógica de predicados monádicos, los cuales pueden ser extendidos a la lógica de relaciones; por otro lado, tal como se muestra en Mosterin (1971), las fórmulas de la lógica de conjuntos son formalizadas con la lógica de relaciones. En consecuencia, el análisis de validez de fórmulas de la lógica de conjuntos también se puede realizar con los árboles de forzamiento para la lógica de relaciones.

El análisis de validez realizado con los árboles de forzamiento semántico para operaciones entre conjuntos, evita el paso por los árboles de forzamiento para la lógica de relaciones, por lo que las pruebas son más cortas y más intuitivas.
Desde el punto de vista didáctico, la simplicidad de las reglas para el forzamiento de marcas, hacen de los árboles de forzamiento para operaciones entre conjuntos, una herramienta de trabajo muy útil.

\section{REFERENCIAS}

- Barrero, T. y Carnielli, W. 2005. Tableaux sin refutación. Matemáticas: Enseñanza Universitaria. 13(2):81-99.

- Beth, E. 1962. Formal methods, an introduction to symbolic logic and to the study of effective operations in arithmetic and logic. Reidel Publishing, Dordrecht, $168 \mathrm{p}$.

- Caicedo, X. 1990. Elementos de lógica y calculabilidad. Bogotá: Una empresa docente.

- Carnielli, W. 1987. Systematization of finite many-valued logics through the method of tableaux. The Journal of Symbolic Logic. 52(2):473-493.

- Mosterín, J. 1971. Teoría axiomática de conjuntos. Barcelona: ediciones Ariel.

- Oubiña, L. 1965. Introducción a la teoría de conjuntos. Buenos Aires: editorial universitaria de Buenos Aires.

- Smullyan, R. 1968. First order logic. Springer-Verlag, Berlin, 158p.

- Sierra, M. 2001. Arboles de forzamiento semántico. Revista Universidad EAFIT. 37(123):53-72.

- Sierra, M. 2006. Caracterización deductiva de los árboles de forzamiento semántico. Revista Ingeniería y Ciencia. 2(3):73-102. 